\title{
Learning curve and complications of minimally invasive transforaminal lumbar interbody fusion
}

\author{
*Pedro S. Silva, M.D., Paulo Pereira, M.D., Pedro Monteiro, M.D., \\ Pedro A. Silva, M.D., and Rui Vaz, Ph.D. \\ Neurosurgery Department, Hospital São João, Porto, Portugal
}

\begin{abstract}
Object. Minimally invasive transforaminal lumbar interbody fusion (MI-TLIF) has the potential advantage of minimizing soft-tissue damage and reducing recovery time compared to open procedures. A steep learning curve has been described for the technique. The aim of the present study was to define the learning curve that describes the progress of a single surgeon performing the MI-TLIF.

Methods. One hundred fifty consecutive patients with degenerative lumbar disease who underwent 1- or 2-level MI-TLIF were included in the study. Operative time, corrected operative time per level, and complications were analyzed. The learning curve was assessed using a negative exponential curve-fit regression analysis.

Results. One hundred ten patients underwent 1-level and 18 patients underwent 2-level MI-TLIF; the remaining 22 underwent a single-level procedure plus an ancillary procedure (decompression at adjacent level, vertebral augmentation through fenestrated pedicle screws, interspinous device at adjacent level). Negative exponential curves appropriately described the relationship between operative time and experience for 1-level surgery and after correction of operative time per level $\left(\mathrm{R}^{2}=0.65\right.$ and 0.57$)$. The median operative time was 140 minutes (interquartile range 120-173 minutes), and a 50\% learning milestone was achieved at Case 12; a 90\% learning milestone was achieved at Case 39. No patient required transfusion in the perioperative period. The overall complication rate was $12.67 \%$ and the most frequent complication was a dural tear (5.32\%). Before the $50 \%$ and $90 \%$ learning milestones, the complication rates were $33 \%$ and $20.51 \%$, respectively.

Conclusions. The MI-TLIF is a reliable and effective option for lumbar arthrodesis. According to the present study, $90 \%$ of the learning curve can be achieved at around the 40th case.

(http://thejns.org/doi/abs/10.3171/2013.5.FOCUS13157)
\end{abstract}

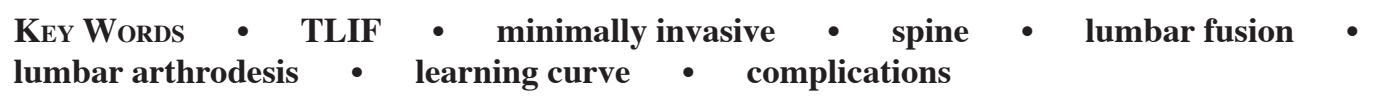

A MONG the available options for the treatment of lumbar degenerative disease, TLIF by MI surgery has been described as advantageous compared with the standard open techniques for lumbar arthrodesis. $^{7}$ The potential benefit of the MI access arises from the reduction of iatrogenic soft tissue and muscle injury. ${ }^{8}$ Single-level TLIF is by far the most common MI fusion procedure. The unilateral transforaminal access provides adequate exposure of the intervertebral disc with minimal neural retraction while the contralateral bony structures and supporting midline ligaments are preserved. ${ }^{6}$

With MI techniques, blood loss is decreased and hospital stay is shorter than with open TLIF.,14,17,19-21 In most studies postoperative pain is reported to be less when using an MI approach. ${ }^{1,4,7,21}$ Additionally, fusion, complication rates, and long-term outcomes seem to be similar between the 2 approaches. ${ }^{1,2,9,11-13,22}$

\footnotetext{
Abbreviations used in this paper: $\mathrm{IQR}=$ interquartile range; $\mathrm{MI}=$ minimally invasive; TLIF = transforaminal lumbar interbody fusion

* Drs. Silva and Pereira contributed equally to this work.
}

One disadvantage to the MI-TLIF is the longer operative time, ${ }^{7}$ at least for the initial cases. The operative times and complications depend on the experience of the surgeon, and a steep learning curve has been described for the technique initiation. ${ }^{10,16}$ The aim of the present study is the definition of the learning curve that describes the progress of a single surgeon performing MI-TLIF. For this purpose, we analyzed the operative time per level and the incidence of complications.

\section{Methods}

We retrospectively analyzed data obtained in patients who underwent MI-TLIF at 2 institutions in the last 6 years. All surgeries were performed by the same senior surgeon (P.P.). We included MI-TLIFs of 1-2 levels for degenerative spondylolisthesis, isthmic spondylolisthesis, recurrent lumbar disc herniation, degenerative lumbar disc disease, or segmental instability.

The operative time and peri- and postoperative complications were assessed by reviewing clinical records for 
each patient. The patients were divided in 3 groups based on the number of levels or an additional surgical procedure performed: Group A comprised 1-level MI-TLIFs; Group B, 2-level MI-TLIFs; and Group C, 1-level MITLIFs plus an ancillary MI procedure (decompression at an adjacent level, vertebral augmentation through fenestrated pedicle screws, or placement of an interspinous device at an adjacent level). For the patients in Groups B and $\mathrm{C}$, the corrected operative time was calculated by dividing the mean operative time of Groups $\mathrm{B}$ and $\mathrm{C}$ by the mean operative time of Group A, yielding a coefficient for correction of the operative time for patients in Groups $\mathrm{B}$ and $\mathrm{C}$. The cases were chronologically ordered and the operative times were plotted on a graph.

The learning curve was assessed by using a negative exponential curve-fit regression analysis $\left(y=\mathrm{ae}^{-\mathrm{bx}}+\mathrm{c}\right)$. In this model (Fig. 1), $y$ is the operative time, $x$ is the experience determined by the number of cases, a is the potential improvement, a $+\mathrm{c}$ defines the beginner's proficiency level, c reflects the expert's proficiency level; $\mathrm{a}+\mathrm{c}$ decreases to $c$ with constant rate of $b$. Thereby, it is possible to calculate the number of prior procedures for which the model predicts a percentage of potential reduction in operative time. These learning milestones are usually defined as $\mathrm{X} \%$ learning milestone in the following: $-\ln [(1$ - X\%)] $/ \mathrm{b}^{3}$ (Fig. 1). The complications were analyzed by assessing their relation to the learning curve. The statistic analysis was performed with GraphPad Prism version 5.0 (GraphPad Software).

The surgical technique has been described previously. ${ }^{6}$ The following adaptations for single-level MI-TLIF were introduced in the present series: the placement of the K-wire in the pedicles bilaterally was performed first; percutaneous transpedicular fixation (CD Horizon, Sextant II; Medtronic Sofamor Danek) was then performed on the side of the patient exhibiting the fewest symptoms. On the contralateral side, the Quadrant system (Medtronic Sofamor Danek) was placed and a facetectomy was performed. Ipsilateral exiting and traversing nerve roots were decompressed as needed. When necessary, the tubular retractor was tilted to the contralateral side, uni- or bilateral flavectomy was performed, and the contralateral traversing nerve root was decompressed on

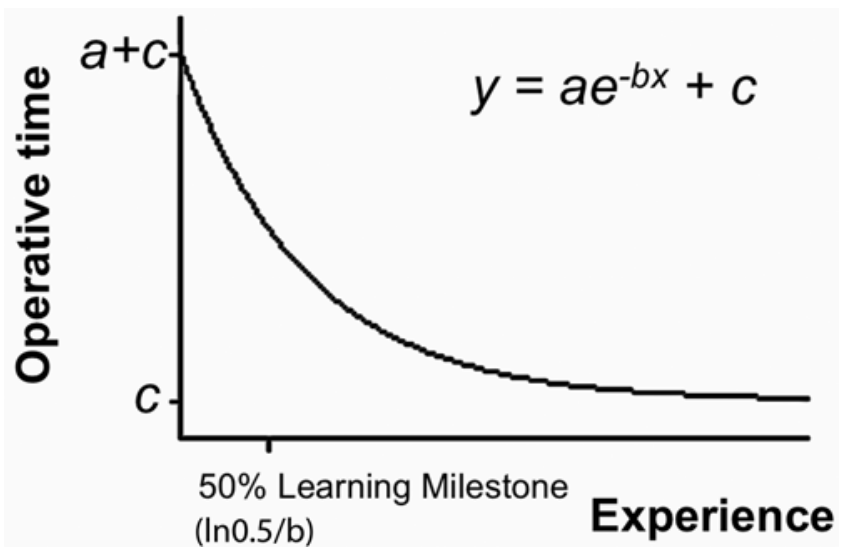

FIG. 1. Graph illustrating the negative exponential model used to describe the learning curves. the lateral recess if needed. After discectomy and bony endplates preparation, autograft (local bone from facet removal) was placed anteriorly in the disc space and inside a Capstone cage (Medtronic Sofamor Danek), which was obliquely impacted in the disc space.

Similarly, for 2-level MI-TLIF, all K-wires were first placed in the pedicles, and percutaneous transpedicular fixation was then performed on 1 side. An MI-TLIF was performed contralaterally at 1 level, and then the tubular retractor was tilted to the adjacent level for repeating the same procedure.

In the Group $\mathrm{C}$ patients, MI-TLIF was performed with one of the aforementioned ancillary MI procedures, according to the respective technique.

\section{Results}

The study was based on 150 patients who underwent MI-TLIF in the last 6 years (Table 1). The average patient age at the time of surgery was 57 years (range 24-82 years) and the series group included 99 women $(66 \%)$ and 51 men (34\%). The mean length of follow-up was 33 months (range 6-74 months). The indications for interbody fusion (Table 2) included degenerative spondylolisthesis in 87 patients (58\%) (Fig. 2), isthmic spondylolisthesis in $29(19.3 \%)$, recurrent lumbar disc herniation in $23(15.3 \%)$, degenerative disc disease in $9(6 \%)$, and segmental instability in $2(1.3 \%)$. One-level surgery was performed in 110 patients (73.3\%; Group A), 2-level TLIF in 18 patients (12\%; Group B), and 1-level TLIF plus an ancillary MI procedure (decompression at adjacent level [13 patients], vertebral augmentation through fenestrated pedicle screws [5 cases], or insertion of an interspinous device at adjacent level [4 patients]) in 22 patients (14.6\%; Group C) (Table 3).

The number of procedures performed per year increased substantially over time (Fig. 3), with 11 surgeries done in the 1st year and 36 in the last year (this number stabilized in the last 3 years at around 35 procedures per year).

The median operative time for patients in Group A

TABLE 1: Patient characteristics

\begin{tabular}{lc}
\hline Characteristic & Value \\
\hline no. of patients & 150 \\
age (yrs) & 57 \\
mean & $24-82$ \\
range & \\
sex (\%) & 66 \\
F & 34 \\
M & \\
follow-up (mos) & 33 \\
mean & $6-74$ \\
range & \\
hospital stay (days) & 3 \\
median & $3-5$ \\
IQR &
\end{tabular}


TABLE 2: Summary of indications for surgery

\begin{tabular}{lc}
\hline \multicolumn{1}{c}{ Indication } & No. of Patients (\%) \\
\hline degenerative spondylolisthesis & $87(58)$ \\
isthmic spondylolisthesis & $29(19.3)$ \\
recurrent lumbar disc herniation & $23(15.3)$ \\
degenerative disc disease & $9(6)$ \\
segmental instability & $2(1.3)$ \\
\hline
\end{tabular}

was 140 minutes (IQR 115-175 minutes; mean 150 minutes). Operative time required for 1-level MI-TLIF decreased significantly as the case number progressed (y $\left.=214 \mathrm{e}^{-0.0758 x}+125.5 ; \mathrm{R}^{2}=0.65\right)($ Fig. 4). For Group A, a $50 \%$ learning milestone was achieved at Case 9 and a $90 \%$ learning milestone at Case 30 . For Group B, the median operative time was 245 minutes (IQR 213-260 minutes; mean 248 minutes), corresponding to a coefficient of 1.65 comparing to Group A. For Group $\mathrm{C}$ the median operative time was 195 minutes (IQR 165-240 minutes; mean 202 minutes), with a coefficient of 1.35 regarding Group A.

The same negative exponential model was applied for the 3 groups taken altogether, after correction of the operative times in Groups B and $\mathrm{C}$ as described above $\left(y=184.7 \mathrm{e}^{-0.059 x}+130 ; \mathrm{R}^{2}=0.57\right)($ Fig. 5). The median operative time for the 150 patients (with corrected values) was 140 minutes (IQR 120-173 minutes). Half of the improvement was expected after 12 cases $(50 \%$ learning milestone), and $90 \%$ of the improvement was expected after 39 cases (90\% learning milestone).

The median time for hospital stay was 3 days (IQR 3-5 days). Complications occurred in 19 cases (12.67\%) and are summarized in Table 4 . The most frequent complication (8 cases [5.32\%]) was a dural tear that was intraoperatively corrected without any consequence in the postoperative period, except in 1 patient with severe spinal stenosis who had a large dural tear with nerve root exteriorization; in this case it was necessary to convert the MI procedure to an open procedure. This patient presented in the postoperative period with persistent neuro- genic bladder and perineal hypesthesia. Before the $50 \%$ and $90 \%$ learning milestones (at Case 12 and Case 39) the complication rates were $33 \%$ and $20.51 \%$, respectively. This evolution is illustrated in Fig. 6. There were 13 patients with complications in Group A (11.8\%). In this single-level group, the complication rate before the $90 \%$ learning milestone (at Case 30) was 23.33\%. There was no chronological difference in severe complications: the cases with screw malposition and extradural hematoma occurred early in the curve (Cases 2 and 23); the cases of neurogenic bladder and meningitis occurred later (Cases 116 and 126). No patient needed blood transfusion during the surgery or in the postoperative period. No patient had clinical complaints or required reoperation directly because of pseudarthrosis.

\section{Discussion}

The MI approaches for lumbar fusion have been reported to be advantageous for minimizing soft-tissue damage and reducing recovery time compared with open surgery. ${ }^{5}$ However, adoption of the technique can be difficult, with a steep learning curve ${ }^{16}$ that can be discouraging and may raise some resistance from the surgeon who is used to an open procedure, with good outcome and few complications. The benefits of MI-TLIF are well documented in the literature. In addition to the advantages of minimal neural retraction and contralateral anatomy preservation, the shorter hospital stay and minimized postoperative pain may allow a patient's earlier return to normal activity. ${ }^{18,22}$ These aspects may also be reflected in economic benefits, saving resources and costs related to spine care. ${ }^{11}$

The major features associated with a learning curve for the surgery are the operative time and complications..$^{15}$ The present study demonstrates how these variables can be considerably improved and stabilized after an initial phase of a few dozen cases, sustaining MI-TLIF as a reliable option for lumbar fusion.

From the statistical methods used to assess learning in health technologies, fitting a line to the data is still
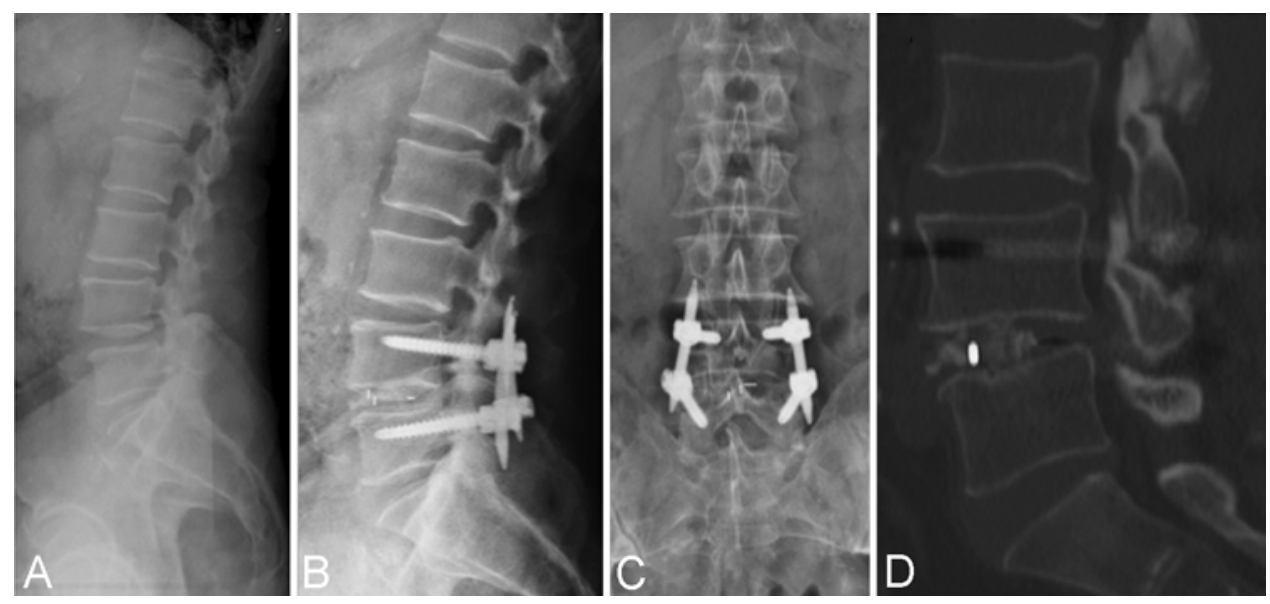

FIG. 2. Illustrative case of a patient with Grade 1 (Meyerding) degenerative spondylolisthesis at L4-5 level who underwent 1-level MI-TLIF. Preoperative radiograph (A). One-year lumbar radiographs (B and C), and lumbar CT scan (D) demonstrate the spondylolisthesis reduction and interbody fusion. The patient's right radicular pain resolved with good functional outcome. 
TABLE 3: Group characteristics*

\begin{tabular}{lllcc}
\hline Characteristic & Group A & Group B & Group C & Total \\
\hline no. of patients (\%) & $110(73.3)$ & $18(12)$ & $22(14.6)$ & 150 \\
op time (mins) & & & & \\
median & 140 & 245 & 195 & 140 \\
IQR & $115-175$ & $213-260$ & $165-240$ & $120-173$ \\
complications (\%) & $13(11.8)$ & $1(0.5)$ & $5(22.7)$ & $19(12.7)$ \\
\hline
\end{tabular}

* Summary of treatments: Group A = 1-level MI-TLIF; Group B = 2-level MI-TLIF; Group C = 1-level MI-TLIF plus ancillary MI procedure.

uncommon. The curve-fitting procedures were only used in $9 \%$ of the studies with that objective..$^{15}$ Furthermore, there are multiple mathematical equations to describe the learning curves, with different shapes and distinctive features..$^{15}$ The negative exponential function has attractive qualities for the description of technique learning. The intuitive shape of the curve reflects the improvement in outcome occurring more rapidly early (that is, in the beginning the curve declines very fast), and the progress in experience continues toward an asymptote. ${ }^{3}$ After we plotted operative times on a graph, we observed that data follow the shape described by a negative exponential equation (Figs. 1, 3, and 4). In the curves constructed from the operative time, we found that an adequate fit to curve was achieved with an $\mathrm{R}^{2}$ of 0.65 for 1-level surgeries and an $\mathrm{R}^{2}$ of 0.57 for all patients after correction of operative times.

From the variable $b$ of the function, which reflects the improvement rate with experience, we determined learning milestones for $50 \%$ and $90 \%$ of the expertise level. The 50\% milestone was achieved relatively fast for single-level surgery (at Case 9) and for the complete series (at Case 12). The 90\% learning milestone determined for Group A (at Case 30), and for the entire series (at Case 39), shows that after these cases the operative time improvement is residual through an asymptote. In the 1-level MI-TLIF, the asymptote is about 126 minutes and in the complete series this value is 130 minutes.

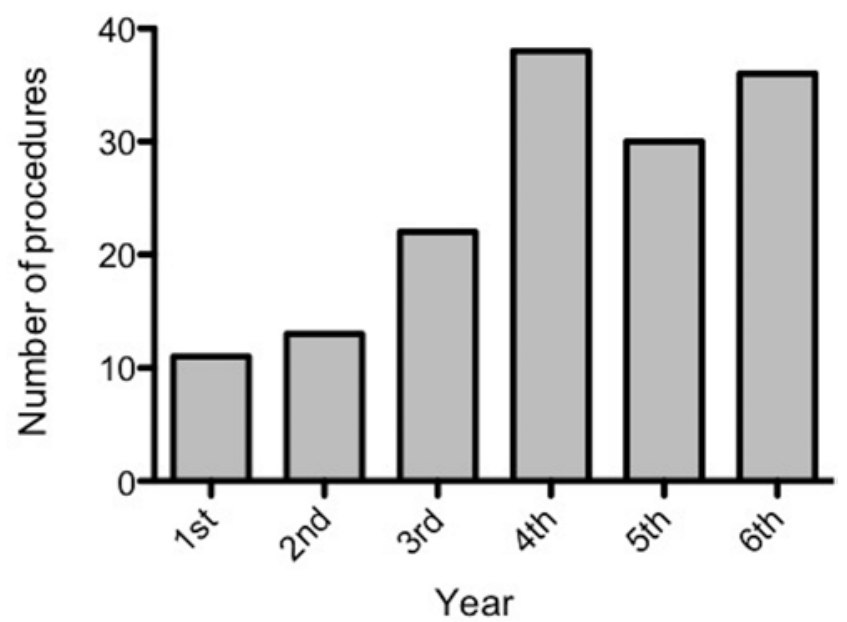

FIG. 3. Bar graph showing the number of procedures performed per year.
Lee et al.$^{10}$ analyzed 86 consecutive patients with degenerative lumbar disease treated by MI-TLIF with the objective of defining and analyzing the learning curve. They used an analog method with corrected operative times per level and a logarithmic curve-fit regression analysis with an $\mathrm{R}^{2}$ of 0.498 for the complete series and 0.622 for a single level. They likewise found that operative time could reach an asymptote after about 30 cases. Both aspects have the same magnitude of those in our fit regression analysis.

The mean operative time for 1-level MI-TLIF varies in several reports from 104 to 389.7 minutes..$^{18}$ In our study the median operative time for the 150 patients (with corrected values) was 140 minutes (IQR 120-173 minutes), which fits in the lower part of the spectrum described. This median operative time after correction is very close to the determined asymptote (130 minutes), reflecting the expected residual improvement after the $90 \%$ learning milestone. The same consideration is valid for the singlelevel series (73.3\% of the procedures), because in these patients the IQR does not differ significantly from that of the total series.

Regarding the complications, we found that results in previous reports were quite variable, with the incidence of complications ranging from $0 \%$ to $33.3 \%$ for MI-TLIF

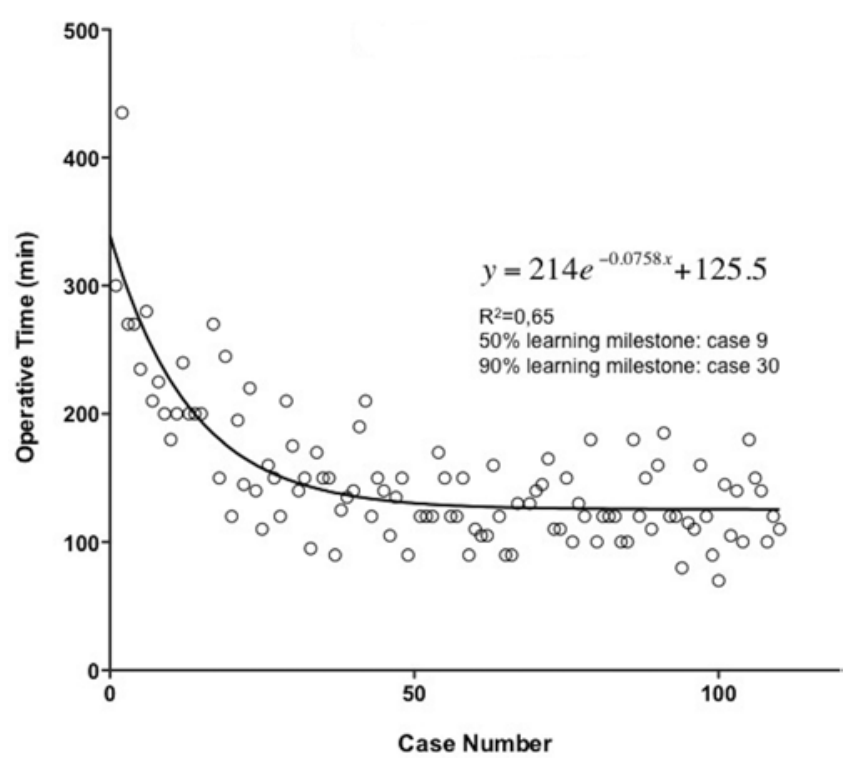

FIG. 4. Learning curve for single-level MI-TLIF as shown by operative time. 


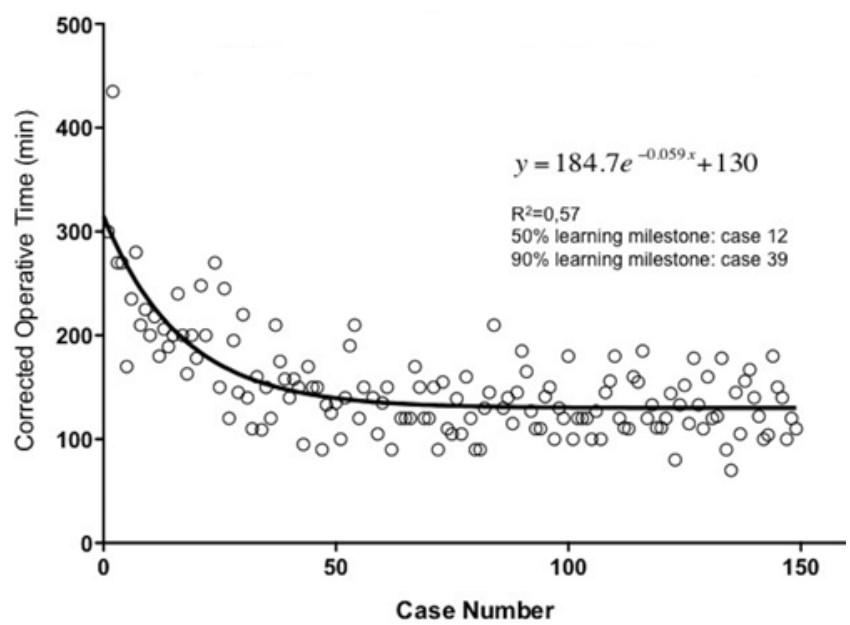

FIG. 5. Learning curve for total series as shown by corrected operative time.

and from $1.6 \%$ to $16.7 \%$ for open TLIF/posterior lumbar interbody fusion. ${ }^{18}$ In the present study, the complication rate was $12.67 \%(11.82 \%$ in Group $\mathrm{A})$, which fits in the range of the data available for the 2 approaches. It is noteworthy that in Group C we had 5 complications in 22 patients. It is possible that for this group the learning curve still did not reach the $90 \%$ improvement, but we cannot also ignore that this group includes more complex procedures that can have a greater probability of complications.

It was demonstrated that the distribution of the complications over time reflects the learning curve, since the complication rates were higher before the learning milestones (33\% and $20.51 \%$ before the $50 \%$ and $90 \%$ learning milestones, respectively).

\section{Conclusions}

The MI-TLIF technique is a reliable and effective option for lumbar arthrodesis. Half of the improvement in the learning can be accomplished after 12 cases and $90 \%$ of the expert's proficiency level is achieved at around 40 cases.

\section{TABLE 4: Summary of complications}

\begin{tabular}{lcc}
\hline \multicolumn{1}{c}{ Complication } & $\begin{array}{c}\text { No. of } \\
\text { Patients }\end{array}$ & Group (no.) \\
\hline $\begin{array}{l}\text { dural tears } \\
\text { persistent neurogenic bladder, peri- } \\
\quad \text { neal hypesthesia* }\end{array}$ & 8 & A (4), B (1), C (3) \\
severe postop sciatica (transient) & 3 & C \\
superficial wound infection & 2 & A \\
deep wound infection \& meningitis & 1 & A, C \\
persistent motor radiculopathy & 1 & A \\
screw malposition requiring revision & 1 & A \\
extradural hematoma (reintervention) & 1 & A \\
myocardial infarction & 1 & A \\
total & 19 & A (13), B (1), C (5) \\
\hline
\end{tabular}

* Occurred in 1 of the 8 patients with a dural tear.

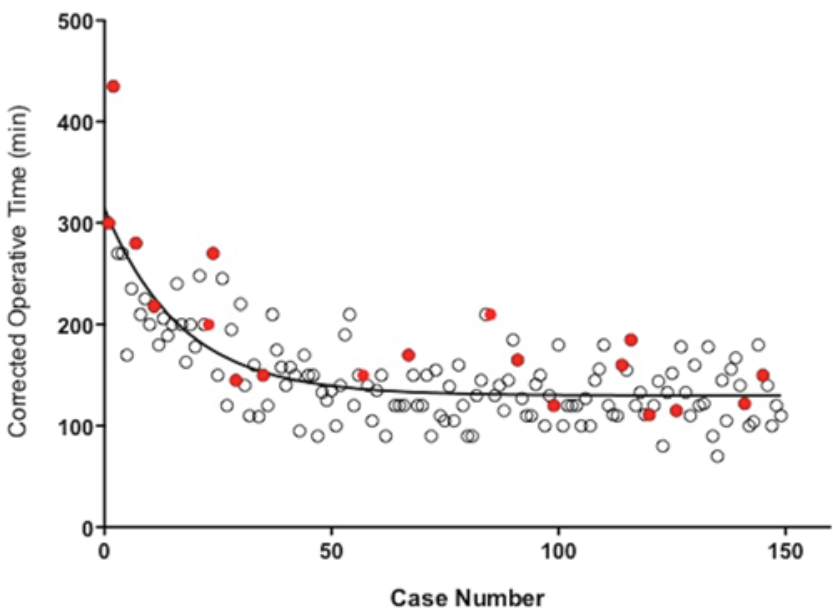

FIG. 6. Complications distribution (red) across the learning curve for entire series.

\section{Disclosure}

The authors report no conflict of interest concerning the materials or methods used in this study or the findings specified in this paper.

Author contributions to the study and manuscript preparation include the following. Conception and design: PS Silva, Pereira, Monteiro. Acquisition of data: PS Silva, Pereira, Monteiro, PA Silva. Analysis and interpretation of data: PS Silva, Pereira, Monteiro. Drafting the article: PS Silva, Pereira. Critically revising the article: Pereira, Vaz. Reviewed submitted version of manuscript: all authors. Approved the final version of the manuscript on behalf of all authors: PS Silva. Statistical analysis: PS Silva. Study supervision: Pereira, Vaz.

\section{References}

1. Adogwa O, Parker SL, Bydon A, Cheng J, McGirt MJ: Comparative effectiveness of minimally invasive versus open transforaminal lumbar interbody fusion: 2-year assessment of narcotic use, return to work, disability, and quality of life. J Spinal Disord Tech 24:479-484, 2011

2. Bagan B, Patel N, Deutsch H, Harrop J, Sharan A, Vaccaro AR, et al: Perioperative complications of minimally invasive surgery (MIS): comparison of MIS and open interbody fusion techniques. Surg Technol Int 17:281-286, 2008

3. Danford DA, Kugler JD, Deal B, Case C, Friedman RA, Saul JP, et al: The learning curve for radiofrequency ablation of tachyarrhythmias in pediatric patients. Am J Cardiol 75:587-590, 1995

4. Dhall SS, Wang MY, Mummaneni PV: Clinical and radiographic comparison of mini-open transforaminal lumbar interbody fusion with open transforaminal lumbar interbody fusion in 42 patients with long-term follow-up. Clinical article. J Neurosurg Spine 9:560-565, 2008

5. Fan S, Hu Z, Zhao F, Zhao X, Huang Y, Fang X: Multifidus muscle changes and clinical effects of one-level posterior lumbar interbody fusion: minimally invasive procedure versus conventional open approach. Eur Spine J 19:316-324, 2010

6. Foley KT, Holly LT, Schwender JD: Minimally invasive lumbar fusion. Spine (Phila Pa 1976) 28 (15 Suppl):S26-S35, 2003

7. Karikari IO, Isaacs RE: Minimally invasive transforaminal lumbar interbody fusion: a review of techniques and outcomes. Spine (Phila Pa 1976) 35 (26 Suppl):S294-S301, 2010

8. Kim CW: Scientific basis of minimally invasive spine surgery: prevention of multifidus muscle injury during posterior lumbar surgery. Spine (Phila Pa 1976) 35 (26 Suppl):S281S286, 2010 
9. Kim JS, Jung B, Lee SH: Instrumented minimally invasive spinal-transforaminal lumbar interbody fusion (MIS-TLIF); minimum 5-years follow-up with clinical and radiologic outcomes. J Spinal Disord Tech [epub ahead of print], 2012

10. Lee JC, Jang HD, Shin BJ: Learning curve and clinical outcomes of minimally invasive transforaminal lumbar interbody fusion: our experience in 86 consecutive cases. Spine (Phila Pa 1976) 37:1548-1557, 2012

11. McGirt MJ, Parker SL, Lerner J, Engelhart L, Knight T, Wang MY: Comparative analysis of perioperative surgical site infection after minimally invasive versus open posterior/transforaminal lumbar interbody fusion: analysis of hospital billing and discharge data from 5170 patients. Clinical article. J Neurosurg Spine 14:771-778, 2011

12. Ntoukas V, Müller A: Minimally invasive approach versus traditional open approach for one level posterior lumbar interbody fusion. Minim Invasive Neurosurg 53:21-24, 2010

13. Parker SL, Adogwa O, Witham TF, Aaronson OS, Cheng J, McGirt MJ: Post-operative infection after minimally invasive versus open transforaminal lumbar interbody fusion (TLIF): literature review and cost analysis. Minim Invasive Neurosurg 54:33-37, 2011

14. Peng CW, Yue WM, Poh SY, Yeo W, Tan SB: Clinical and radiological outcomes of minimally invasive versus open transforaminal lu mbar interbody fusion. Spine (Phila Pa 1976) 34: 1385-1389, 2009

15. Ramsay CR, Grant AM, Wallace SA, Garthwaite PH, Monk AF, Russell IT: Statistical assessment of the learning curves of health technologies. Health Technol Assess 5:1-79, 2001

16. Schizas C, Tzinieris N, Tsiridis E, Kosmopoulos V: Minimally invasive versus open transforaminal lumbar interbody fusion: evaluating initial experience. Int Orthop 33:1683-1688, 2009
17. Shunwu F, Xing Z, Fengdong Z, Xiangqian F: Minimally invasive transforaminal lumbar interbody fusion for the treatment of degenerative lumbar diseases. Spine (Phila Pa 1976) 35:1615-1620, 2010

18. Tian NF, Wu YS, Zhang XL, Xu HZ, Chi YL, Mao FM: Minimally invasive versus open transforaminal lumbar interbody fusion: a meta-analysis based on the current evidence. Eur Spine J [epub ahead of print], 2013

19. Villavicencio AT, Burneikiene S, Roeca CM, Nelson EL, Mason A: Minimally invasive versus open transforaminal lumbar interbody fusion. Surg Neurol Int 1:12, 2010

20. Wang J, Zhou Y, Zhang ZF, Li CQ, Zheng WJ, Liu J: Comparison of one-level minimally invasive and open transforaminal lumbar interbody fusion in degenerative and isthmic spondylolisthesis grades 1 and 2. Eur Spine J 19:1780-1784, 2010

21. Wang J, Zhou Y, Zhang ZF, Li CQ, Zheng WJ, Liu J: Minimally invasive or open transforaminal lumbar interbody fusion as revision surgery for patients previously treated by open discectomy and decompression of the lumbar spine. Eur Spine J 20:623-628, 2011

22. Wu RH, Fraser JF, Hartl R: Minimal access versus open transforaminal lumbar interbody fusion: meta-analysis of fusion rates. Spine (Phila Pa 1976) 35:2273-2281, 2010

Manuscript submitted April 15, 2013.

Accepted May 29, 2013.

Please include this information when citing this paper: DOI: 10.3171/2013.5.FOCUS13157.

Address correspondence to: Pedro S. Silva, M.D., Hospital São João, Neurosurgery Department, 4200319 Porto, Portugal. email: pedrodossantossilva@gmail.com. 